\title{
Development of a Nonlinear Collision Operator for GNET Code*)
}

\author{
Yoshitada MASAOKA and Sadayoshi MURAKAMI \\ Department of Nuclear Engineering, Kyoto University, Nishikyo, Kyoto 615-8530, Japan
}

(Received 29 November 2012 / Accepted 22 April 2013)

\begin{abstract}
A nonlinear collision operator is formulated in the polar coordinate $(v, \cos \theta)$ in order to study the effect of collisions among high-energy particles on their confinements in toroidal plasmas. The Monte Carlo collision operator is derived to be easily implemented to the orbit following code. The nonlinear collision model is benchmarked with the Boozer collision model for the Maxwellian plasma. This collision model enables the effect of self collisions of $\alpha$-particles and energetic particles to be investigated on plasma heating, e.g. NBI and ICRF heating.
\end{abstract}

(C) 2013 The Japan Society of Plasma Science and Nuclear Fusion Research

Keywords: nuclear fusion, $\alpha$-particle confinement, plasma heating, nonlinear collision operator, Monte Carlo method

DOI: $10.1585 /$ pfr.8.2403106

\section{Introduction}

In a D-T fusion plasma $\alpha$ particles $(E=3.5 \mathrm{MeV})$ are generated by the fusion reaction and those $\alpha$ particles mainly collide only with electrons because of very high velocity compared to the thermal ion ones. Thus it is considered that the pitch angle scattering is very small for the $\alpha$ particle during the energy slowing down.

On the other hand, the relative velocity between the $\alpha$ particles sometimes becomes very small. In this case they would experience additional pitch angle scattering. Although the density of high-energy particles is much less than that of thermal other ions, the collisions between $\alpha$ particles would have some effect on pitch angle scattering. It was reported that the nonlinear collisions by each fast $\alpha$ particle has sometimes larger effect than that by other background ions [1].

This nonlinear collision effect may lead to deteriorates the $\alpha$ particle confinement, because of the increase of pitch angle scatterings. Especially, in helical systems, highenergy particle trajectories are complicated due to the three dimensional magnetic configuration and the small increase of pitch angle scattering might affect the high-energy particle confinement significantly.

Therefore, the analysis including the both complicated orbit and the nonlinear collisions are necessary to examine the $\alpha$-particle confinement in toroidal plasmas. The nonlinear collision operator however has not yet been formulated for the orbit following type of Monte Carlo code. In this paper, we formulate the nonlinear collision operator and the Monte Carlo collision operator which can be easily implemented to the orbit following type codes is derived.

author'se-mail: masaoka@p-grp.nucleng.kyoto-u.ac.jp

*) This article is based on the presentation at the 22nd International Toki Conference (ITC22).

\section{The Nonlinear Collision Operator}

The nonlinear collision operator was formulated by Killeen [2] and Karney [3], but this operator is not described in the polar velocity coordinates $(v, \cos \theta)$ and does not consider the multi plasma species, where $\cos \theta=v / v_{\|}$. The change of the pitch angle by Coulomb collisions is generally described in the $(v, \cos \theta)$ space and the actual fusion plasma consists of multi species. Therefore, it is important to formulate the nonlinear collision operator assuming the multi plasma species in the polar coordinates $(v, \cos \theta)$.

We first consider the Fokker-Plank equation, which is useful for the study of plasma kinetic behaviors. This equation can be reduced to an equation in time and two velocity dimensions only.

$$
\begin{aligned}
\frac{\partial f_{a}}{\partial t} & +\boldsymbol{v} \cdot \nabla f_{a}+\frac{q_{a}}{m_{a}}(\boldsymbol{E}+\boldsymbol{v} \times \boldsymbol{B}) \cdot \frac{\partial f_{a}}{\partial \boldsymbol{v}} \\
& =\sum_{b} C\left(f_{a}, f_{b}\right),
\end{aligned}
$$

where $f_{s}$ is the distribution function of particle species $s$. $\boldsymbol{v}$ is a drift velocity, $q_{s}$ and $m_{s}$ are a charge and mass of species s, $\boldsymbol{E}$ is the electric field and $\boldsymbol{B}$ is the magnetic field. $C\left(f_{a}, f_{b}\right)$ is the collision term for species a colliding with species $b$.

Because Coulomb collisions in plasma are typically dominated by small pitch angle scattering, the collision term can be written using the flux as

$$
C\left(f_{a}, f_{b}\right)=-\nabla \cdot \stackrel{\leftrightarrow}{S}_{c}^{a / b},
$$

where $\stackrel{\leftrightarrow}{S}_{c}^{a / b}$ is the collisional flux in velocity space and expressed by Landau collision integral [4] as

$$
\stackrel{\leftrightarrow}{S}_{c}^{a / b}=\frac{q_{a}^{2} q_{b}^{2}}{8 \pi \epsilon_{0}^{2} m_{a}} \ln \Lambda^{a / b},
$$




$$
\int \stackrel{\leftrightarrow}{U}(u) \cdot\left(\frac{f_{a}(v)}{m_{b}} \frac{\partial f_{b}\left(\boldsymbol{v}^{\prime}\right)}{\partial \boldsymbol{v}^{\prime}}-\frac{f_{b}\left(\boldsymbol{v}^{\prime}\right)}{m_{a}} \frac{\partial f_{a}(\boldsymbol{v})}{\partial \boldsymbol{v}}\right) \mathrm{d}^{3} \boldsymbol{v}^{\prime}
$$

where $\epsilon_{0}$ is the dielectric constant in vacuum, $\ln \Lambda^{a / b}$ is the Coulomb logarithm, and

$$
\stackrel{\leftrightarrow}{U}(u)=\frac{u^{2} \stackrel{\leftrightarrow}{I}-\boldsymbol{u u}}{u^{3}}, \quad \boldsymbol{u}=\boldsymbol{v}-\boldsymbol{v}^{\prime}
$$

Equation. (3) is the useful form of the collision operator for analytical study. But, it is not a convenient form for the numerical implementation. Using an equivalent representation in terms of Rosenbluth potentials, we can rewrite the eq. (3). Also applying the slightly more convenient notation of Trubnikov, we define two Rosenbluth potentials [5] as

$$
\begin{aligned}
& \phi_{b}\left(\boldsymbol{v}^{\prime}\right)=-\frac{1}{4 \pi} \int \frac{f_{b}\left(\boldsymbol{v}^{\prime}\right)}{\left|\boldsymbol{v}^{\prime}-\boldsymbol{v}\right|} \mathrm{d}^{3} \boldsymbol{v}^{\prime}, \\
& \psi_{b}\left(\boldsymbol{v}^{\prime}\right)=-\frac{1}{8 \pi} \int\left|\boldsymbol{v}-\boldsymbol{v}^{\prime}\right| f_{b}\left(\boldsymbol{v}^{\prime}\right) \mathrm{d}^{3} \boldsymbol{v}^{\prime} .
\end{aligned}
$$

These satisfy Poisson's equations in velocity space

$$
\begin{aligned}
& \nabla^{2} \phi_{b}(\boldsymbol{v})=f_{b}(\boldsymbol{v}), \\
& \nabla^{2} \psi_{b}(\boldsymbol{v})=\phi_{b}(\boldsymbol{v}) .
\end{aligned}
$$

Thus, $\stackrel{\leftrightarrow}{S}_{c}^{a / b}$ can be expressed in terms of these potentials as

$$
\begin{aligned}
& \stackrel{\leftrightarrow}{S}_{c}^{a / b}=-\stackrel{\leftrightarrow}{D}_{c}^{a / b} \nabla f_{a}(\boldsymbol{v})+\boldsymbol{F}_{c}^{a / b} f_{a}(\boldsymbol{v}), \\
& \stackrel{\leftrightarrow}{D}_{c}^{a / b}=-\frac{4 \pi \Gamma^{a / b}}{n_{b}} \nabla \nabla \psi_{b}(\boldsymbol{v}), \\
& \boldsymbol{F}_{c}^{a / b}=-\frac{4 \pi \Gamma^{a / b}}{n_{b}} \frac{m_{a}}{m_{b}} \nabla \phi_{b}(\boldsymbol{v}),
\end{aligned}
$$

$$
\leftrightarrow a / b
$$

where $\stackrel{\leftrightarrow}{D}_{c}^{a / b}$ and $\boldsymbol{F}_{c}^{a / b}$ are a diffusion tensor and a friction force, respectively, and

$$
\Gamma^{a / b}=\frac{n_{b} q_{a}^{2} q_{b}^{2} \ln \Lambda^{a / b}}{4 \pi \epsilon_{0}^{2} m_{a}^{2}} .
$$

Because of a singular point at $v^{\prime}=v$ it is difficult to calculate $\phi_{b}$ numerically. However, there is a useful method for the calculation of the Rosenbluth potentials, where the distribution function $f$ is expressed by Legendre harmonics $P_{l}(v, \cos \theta)$. The Legendre expansion of the distribution function $f(v, \cos \theta)$ is written as

$$
f(v, \cos \theta)=\sum_{l=0}^{\infty} f^{(l)}(v) P_{l}(\cos \theta),
$$

where

$$
f^{(l)}(v)=\frac{2 l+1}{2} \int_{0}^{\pi} f(v, \cos \theta) P_{l}(\cos \theta) \sin \theta \mathrm{d} \theta .
$$

The Legendre polynomials are evaluated using the recurrence relation [6]

$$
\begin{aligned}
& P_{0}(\mu)=1, \\
& (l+1) P_{l+1}(\mu)=(2 l+1) \mu P_{l}(\mu)-l P_{l-1}(\mu) .
\end{aligned}
$$

Using this decomposing distribution function $f$, the Rosenbluth potentials for each species $b$ are written as

$$
\begin{aligned}
\phi_{b}^{(l)}(v)= & -\frac{1}{2 l+1}\left[\int_{0}^{v} \frac{v^{l+2}}{v^{l+1}} f_{b}^{(l)}\left(v^{\prime}\right) \mathrm{d} v^{\prime}\right. \\
& \left.+\int_{v}^{\infty} \frac{v^{l}}{v^{\prime l-1}} f_{b}^{(l)}\left(v^{\prime}\right) \mathrm{d} v^{\prime}\right], \\
\psi_{b}^{(l)}(v)= & \frac{1}{2\left(4 l^{2}-1\right)} \times \\
& {\left[\int_{0}^{v} \frac{v^{l+2}}{v^{l-1}}\left(1-\frac{\left(l-\frac{1}{2}\right) v^{\prime 2}}{\left(l+\frac{3}{2}\right) v^{2}}\right) f_{b}^{(l)}\left(v^{\prime}\right) \mathrm{d} v^{\prime}\right.} \\
& \left.+\int_{v}^{\infty} \frac{v^{l}}{v^{\prime l-3}}\left(1-\frac{\left(l-\frac{1}{2}\right) v^{2}}{\left(l+\frac{3}{2}\right) v^{\prime 2}}\right) f_{b}^{(l)}\left(v^{\prime}\right) \mathrm{d} v^{\prime}\right] .
\end{aligned}
$$

Thus the total Rosenbluth potentials are written as

$$
\begin{aligned}
& \phi_{a}(v, \lambda)=\sum_{l=0}^{\infty} \sum_{b} \frac{m_{a}+m_{b}}{m_{b}} \phi_{b}^{(l)}(v) P_{l}(\lambda), \\
& \psi_{a}(v, \lambda)=\sum_{l=0}^{\infty} \sum_{b} \psi_{b}^{(l)}(v) P_{l}(\lambda) .
\end{aligned}
$$

In the polar coordinate $(v, \lambda), \nabla \nabla \psi$ and $\nabla \phi$ in Eq. (9) become

$$
\begin{aligned}
(\nabla \nabla \psi)_{v v}= & \frac{\partial^{2} \psi}{\partial v^{2}}, \\
(\nabla \nabla \psi)_{\lambda \lambda}= & \frac{1-\lambda^{2}}{v^{2}}\left(\frac{1-\lambda^{2}}{v^{2}} \frac{\partial^{2} \psi}{\partial \lambda^{2}}\right. \\
& \left.-\frac{\lambda}{v^{2}} \frac{\partial \psi}{\partial \lambda}+\frac{1}{v} \frac{\partial \psi}{\partial v}\right), \\
(\nabla \nabla \psi)_{v \lambda}= & (\nabla \nabla \psi)_{\lambda v} \\
= & -\frac{1-\lambda^{2}}{v^{2}}\left(\frac{\partial^{2} \psi}{\partial v \partial \lambda}-\frac{1}{v} \frac{\partial \psi}{\partial \lambda}\right), \\
(\nabla \phi)_{v}= & \frac{\partial \phi}{\partial v}, \\
(\nabla \phi)_{\lambda}= & \frac{\sqrt{1-\lambda^{2}}}{v} \frac{\partial \phi}{\partial \lambda} .
\end{aligned}
$$

We differentiate partially $\psi$ and $\phi$, and using the relationship of Eq. (13) and Eq. (14) and differentiate the function of $v$ and $\lambda$ individually. And the friction force vector $\boldsymbol{F}_{c}^{a / b}$ and diffusion tensor $\stackrel{\leftrightarrow}{D}_{c}^{a / b}$ become

$$
\begin{aligned}
& \stackrel{\leftrightarrow}{D}_{c}^{a / b}=-\frac{4 \pi \Gamma^{a / b}}{n_{b}}\left(\begin{array}{ll}
(\nabla \nabla \psi)_{v v} & (\nabla \nabla \psi)_{v \lambda} \\
(\nabla \nabla \psi)_{\lambda v} & (\nabla \nabla \psi)_{\lambda \lambda}
\end{array}\right), \\
& \boldsymbol{F}_{c}^{a / b}=-\frac{4 \pi \Gamma^{a / b}}{n_{b}} \frac{m_{a}}{m_{b}}\left(\begin{array}{l}
(\nabla \phi)_{v} \\
(\nabla \phi)_{\lambda}
\end{array}\right) .
\end{aligned}
$$

\section{Monte Carlo Collision Operator}

In order to analyze the confinement of high-energy ions in the presence of collisions, it is convenient to use the the Monte Carlo method. Therefore, it is necessary to derive the Monte Carlo collision operator from FokkerPlanck type equation. 
Monte Carlo operators which represent a random walk in the velocity space $(v, \lambda)$ with a time step $\Delta t$ are now given by changing the values of the invariants $I^{i}$ at the $n$th step to those at the next step $I_{n+1}^{i}$, as follows

$$
I_{n+1}^{i}=I_{n}^{i}+\Delta I^{i}
$$

where the quantitie $\Delta I^{i}$ is the stochastic value. The expectation value (the friction force) and covariance (the diffusion) of them are given by

$$
\begin{aligned}
& E\left(\Delta I^{i}\right)=\left[\boldsymbol{F}_{c}^{a / b, i}+\frac{1}{g} \frac{\partial}{\partial I_{j}}\left(g \stackrel{\leftrightarrow}{D}_{c}^{a / b, i j}\right)\right] \Delta t, \\
& C\left(\Delta I^{i}, \Delta I^{j}\right)=2 \stackrel{\leftrightarrow}{D}_{c}^{a / b, i j} \Delta t,
\end{aligned}
$$

where $g=v^{2}$.

In general, the covariance matrix $C\left(\Delta I^{i}, \Delta I^{j}\right)$ is symmetric and positive definite, and can be diagonalized, i.e, a unitary matrix $M$ such as

$$
C\left(\Delta I^{i}, \Delta I^{j}\right)=M^{i k} \Lambda^{k l} M^{j l},
$$

exists, where $\Lambda^{k l}=\operatorname{diag}\left(\Lambda_{1}, \Lambda_{2}\right)$ is the diagonal matrix of the eigenvalues. Because these eigenvalues of matrix $C$ are positive, we introduce the matrix $L^{k l}=\operatorname{diag}\left(\sqrt{\Lambda_{1}}, \sqrt{\Lambda_{2}}\right)$, which write the covariance matrix as

$$
C\left(\Delta I^{i}, \Delta I^{j}\right)=M^{i k} L^{k m} L^{l m} M^{j l} .
$$

From this relation, as well as Eqs. (25) and (26), $\Delta I^{i}$ is expressed as

$$
\Delta I^{i}=E\left(\Delta I^{i}\right)+M^{i k} L^{k l} \xi^{l},
$$

where $\xi^{l}$ is $\xi= \pm 1$ and this sign is chosen at random. This completes the construction of the Monte Carlo operator. The specific velocity and pitch angle scattering are described as, respectively,

$$
\begin{aligned}
& \Delta v=E(\Delta v)+M^{v v} L^{v 1} \xi^{1}+M^{v \lambda} L^{\lambda 2} \xi^{2}, \\
& \Delta \lambda=E(\Delta \lambda)+M^{\lambda v} L^{v 1} \xi^{1}+M^{\lambda \lambda} L^{\lambda 2} \xi^{2} .
\end{aligned}
$$

And the energy scatting can be obtained by Eq. (30),

$$
\begin{aligned}
\Delta E= & m_{a} v_{0}\left\{E(\Delta v)+\left(M^{v v} L^{v}\right)^{2}+\left(M^{v \lambda} L^{\lambda}\right)^{2}\right. \\
& \left.+M^{v v} L^{v 1} \xi^{1}+M^{v \lambda} L^{\lambda 2} \xi^{2}\right\} .
\end{aligned}
$$

In the case of maxwellian $f_{b}$, this nonlinear collision operator corresponds to the Boozer collision operator [7]:

$$
\begin{aligned}
\Delta \lambda= & -\lambda_{0} v_{0} \Delta t \pm \sqrt{\left(1-\lambda_{0}^{2}\right) v_{0} \Delta t}, \\
\Delta E= & -2 v_{E} \Delta t\left\{E_{0}-\left(\frac{3}{2}+\frac{E_{0}}{v_{E}} \frac{\mathrm{d} v_{E}}{\mathrm{~d} E}\right) T_{b}\right\} \\
& \pm \sqrt{T_{b} E_{0} v_{E} \Delta t} .
\end{aligned}
$$

\section{Benchmark}

In order to compare the nonlinear collision model with the Booezer collision model, we consider the drift kinetic

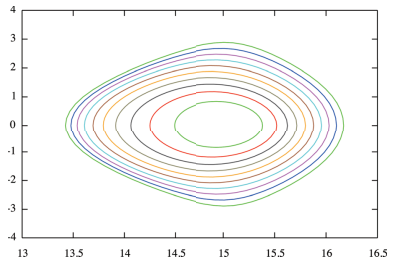

(a) The magnetic configuration

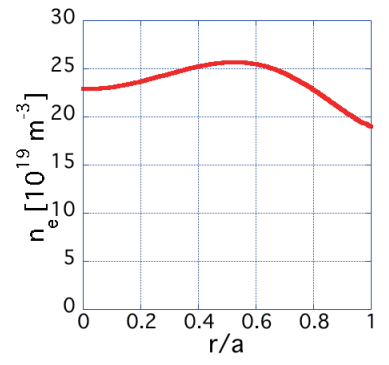

(c) The plasma density

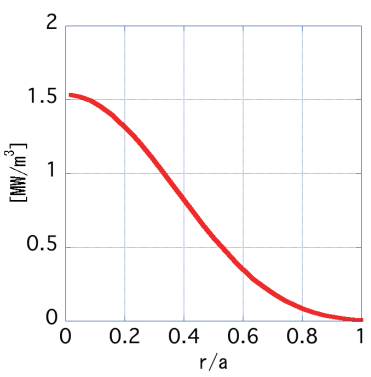

(b) The fusion power



(d) The plasma temperature
Fig. 1 The magnetic configuration of FFHR-d1: The horizontal axis is a plasma major axis, and the longitudinal axis is a $Z$ direction. The Fusion power profile, plasma density profile, and plasma temperature profile.

equation for the $\alpha$ particles in five-dimension phase space, with pitch angle and energy scattering;

$$
\begin{aligned}
\frac{\partial f}{\partial t} & +\left(\boldsymbol{v}_{\|}+\boldsymbol{v}_{D}\right) \cdot \nabla f+\dot{\boldsymbol{v}} \cdot \nabla_{V} f \\
& =C^{\text {coll }}(f)+L^{\text {particle }}(f)+S_{\alpha},
\end{aligned}
$$

where $f$ is the distribution function of $\alpha$ particles. $\boldsymbol{v}_{\|}$and $\boldsymbol{v}_{D}$ are the velocity parallel to magnetic line and the drift velocity respectively. $L^{\text {particle }}$ is the loss term due to the escaping particle from the last closed flux surface (LCFS). $S_{\alpha}$ is the source term of the $\alpha$ particle generated by the fusion reaction. $C^{\text {coll }}$ is a linear Coulomb collision operator [7] or this nonlinear collision operator. In this paper, we use GNET code in the FFHR-d1 $[8,9]$ configuration without orbit calculation. FFHR-d1 is a helical fusion DEMO reactor and has the magnetic field strength $4.5 \mathrm{~T}$, the finite $\beta_{0}$ value $8.4 \%$. The magnetic configuration, plasma density, temperature profile, and the fusion power profile are shown in Fig. 1. We consider the collision of $\alpha$ particle with Maxwellian electron, deuterium, and tritium.

We run the GNET code [10] until the distribution function of the alpha particles has reached a steady state using the Boozer collision model and the nonlinear collision model. Figure 2 shows the velocity space distributions of alpha particles in the Boozer collision model case and the nonlinear collision model case. $v_{\|}$and $v_{\perp}$ are the velocity parallel and perpendicular to magnetic field, respectively. $v_{\|}$and $v_{\perp}$ are normalized by the velocity of $\alpha$ particle with $1 \mathrm{MeV}, v_{1 \mathrm{Mev}}$. The alpha particles slow down isotropically in the both models and we find that the distri- 


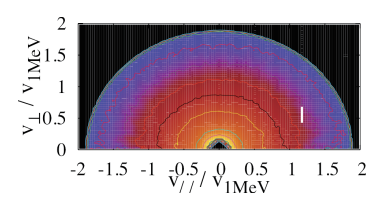

(a) Boozer model

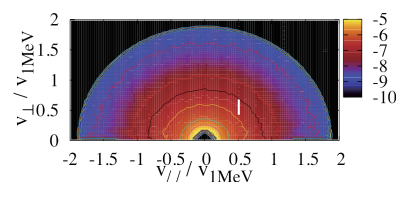

(b) the nonlinear model
Fig. 2 The distribution function based on Boozer model and the nonlinear model: this nonlinear collision model reproduces the alpha particle distribution function of the Boozer collision model.

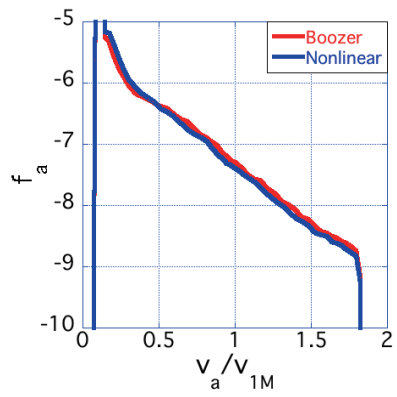

(a) Total

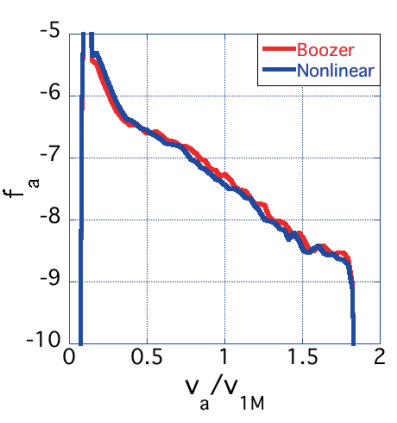

(b) $\mathrm{r} / \mathrm{a}=0.25$

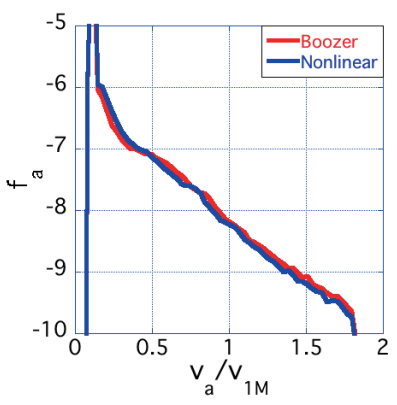

(c) $\mathrm{r} / \mathrm{a}=0.5$

Fig. 3 The distribution function of a pitch angle $60 \%$ at the each radial position and total: For each figures, this nonlinear collision model reproduces the alpha particle distribution function of the Boozer collision model. bution of the Boozer model and the nonlinear model show similar distributions. In order to compare in detail, we plot the velocity space distribution function in the pitch angle $\theta=60 \%$ for total and $r / a=0.25$ and 0.5 (Fig. 3). The red line shows the velocity space distribution in the Boozer collision model case, and the blue one shows the velocity space distribution in the nonlinear collision model case. We can also see that our nonlinear collision model agree well with that of the Boozer one.

\section{Conclusion}

We have formulated the nonlinear collision operator in order to study the collision effect between high energy ions. We have derived the Monte Carlo collision operator to be easily implemented to the orbit following type code such as GNET. Our nonlinear collision model has been benchmarked comparing with the Boozer collision model for Maxwellian fusion plasma. The calculated distributions of the nonlinear collision model agree well with that of the Boozer one. This collision model enables the effect of self collisions of $\alpha$-particles and energetic particles to be investigated on plasma heating, e.g. NBI and ICRF heating.

[1] K. Okano et al., Proc. 28th EPS Conf. Control. Fus. Plas. Phys, pp.809-812 (2001).

[2] J. Killeen et al., Computational Methods for Kinetic Models of Magnetically Confined Plasmas (Springer, New York, 1986).

[3] C.F.F. Karney, Comput. Phys. 4, 183 (1986).

[4] L.D. Landau, Phys. Z Sowjet. 10, 154 (1936).

[5] N. Rosenbluth et al., Phys. Rev. 107, 1 (1957).

[6] M. Abramowitz et al., Handbook of Mathematical Functions (Dover, New York, 1965).

[7] A. Boozer et al., Phys. Fluids 24 (5), 851 (1981).

[8] A. Sagara et al., Fusion Eng. Des. in press.

[9] T. Goto et al., Plasma Fusion Res. 7, 2405084 (2012).

[10] S. Murakami et al., Nucl Fusion 42, L19 (2002). 